\title{
Worldwide VLF Standard Frequency and Time Signal Broadcasting
}

\author{
A. D. Watt, ${ }^{1}$ R. W. Plush, ${ }^{1}$ W. W. Brown, and A. H. Morgan \\ Contribution From the Boulder Laboratories, National Bureau of Standards, Boulder, Colo.
}

(Received June 15, 1961)

\begin{abstract}
Recent studies and measurements have shown that the phase stability of the signals in the VLF region is very much higher than in the HF spectrum. This fact, along with its excellent coverage characteristics, has caused considerable interest in employing this medium for the wide distribution of standard frequencies and time reference. Basic limitations in stability of the received signals are discussed, including path phase distortion, carrier-tonoise and envelope delay variations as related to precise synchronization of clocks, and highly accurate frequency calibrations.

Also included is a discussion of the present services of standard frequency and time signal stations throughout the world at HF, LF, and VLF.
\end{abstract}

\section{Introduction}

The requirement for better standards for precise measurements of frequency and time which are readily available has constantly increased with advances in various fields of science. The scientific unit of time is determined astronomically, and is determined to an uncertainty of a few parts in $10^{9}$ in the course of a year. Since frequency is related inversely to time interval, this uncertainty, strictly speaking, must be transferred to the specification of absolute frequency. Frequency generators, however, stable to parts in $10^{10}$ or $10^{11}$ now exist, and form the basis for relative measurements of frequency which for many purposes satisfy the increased requirements mentioned above.

The history of the development of our present system of time is an extremely fascinating subject and it is interesting to note Newton's definition of time, "Absolute, true and mathematical time, of itself, and by its own nature, flows uniformly on, without regard to anything external," [Mach, 1942]. Most of man's time systems have been based on an attempt to find a reference which "flows uniformly on." The very great influence upon our lives and surroundings caused by the revolution of the earth around the sun and the rotation of the earth on its axis has resulted in various definitions ${ }^{2}$ of time in terms of years (orbital rotation), days (axial rotation), and subdivision of these units in hours, minutes, and seconds. Time based on apparent solar days (from noon to noon) although apparently satisfactory as a time reference for many applications was shown by astronomical observations to have appreciable nonuniformity.

\footnotetext{
${ }^{1}$ Present address of A. D. Watt and R. W. Plush: DECO Electronics, Inc., Boulder Division, 8401 Baseline Road, Boulder, Colo.

2 An interesting and useful description of time and time signals is contained in "United States Naval Observatory Circular No. 49," published by the U.S. Naval Observatory, Washington 25, D.C. (March 8, 1954). See also "Astronomical Time," G. M. Clemence, Rev. Mod. Phys. 29, 1 (January 1957) and "The Way Things Are," P. W. Bridgman, pp. 135-141 (Harvard University Press, Cambridge, Mass., 1959).
}

Since apparent solar days are variable in length with season, mean solar time was devised and the tabulation of this difference between the apparent value and the mean value is called the equation of time. This difference has a maximum value a little in excess of $16 \mathrm{~min}$. Apparent solar days are variable in length with season for two reasons: partly because of the variation in the angular velocity of the sun along the ecliptic in accordance with Kepler's second law of planetary motion, and partly because the inclination of the ecliptic to the celestial equator introduces a variation in the rate at which the projected coordinate of the apparent sun moves along the celestial equator. The mean sun is a fictitious body which moves along the celestial equator at a uniform rate equal to the mean rate of the apparent sun. Because of the fact that a solar time reference (date and time of day, etc.) will depend upon the observer's position, Universal Time (also known as Greenwich mean time) was established with the mean solar time reference based on the prime meridian at Greenwich, England. The problem of relative position for civil purposes has been solved by dividing the earth into various time zones usually differing by one hour with lines of demarcation chosen to minimize the inconvenience caused by crossing time zones in heavily populated areas.

Three kinds of Universal Times are: UT0, which is uncorrected mean solar time; UT1, is Universal Time corrected for observed polar motion and represents the true angular rotation of the earth about its axis: ${ }^{3}$ UT2, is Universal Time corrected for both observed polar motion and seasonal variation in speed of rotation of the earth on an extrapolated basis.

The fundamental unit of time was until very recently the mean solar second which was defined as $\frac{1}{86,400}$ of a mean solar day. This fundamental unit

3 Because of the increased precision with which the earth's rotation can be measured with respect to a reference star rather than the sun, the period of the earth's rotation with respect to the vernal equinox, called a sidereal day, is used for precise determination of the earth's rotation. 
of time based upon the mean solar day appeared to be entirely satisfactory until astronomical observations and advances in the fields of communication, electronic frequency standards, and precision instrumentation showed that in fact the earth was not rotating in a strictly constant manner. Even after the corrections of U'T1 and U'T2, time based on this reference did not flow on uniformly as our definition of it would require. A more uniform astronomical time based on the yearly motion of the earth about the sun, called Ephemeris Time, has long been known. The present definition of the second is $\frac{1}{31,556,925.9747}$ of the tropical year for January 0, 1900 at $12 \mathrm{hr}$ Ephemeris Time [Markowitz, 1959]. This was adopted by the International Committee of Weights and Measures in 1956 and ratified by the 11th General Conference on Weights and Measures in 1960. Discussion of various astronomical and atomic times has been given by Markowitz [1959].

Although quartz crystal clocks were instrumental in revealing many of the variations in rotation of the earth, it was not until atomic standards were placed in use that a new reference of time became conceivable. The use of molecular and atomic spectral lines as frequency standards [Lyons, 1952] has led to the speculation that perhaps here we have a reference which can be made highly independent of external variable influences and as a result may produce a time base of greatly improved uniformity and convenience. The difficulty of keeping molecular and atomic clocks in operation for long periods of time has up until recently prevented their use as a basic time reference. A determination of the atomic transition frequency of cesium in terms of Ephemeris Time by Markowitz, Hall, Essen, and Parry [1958] indicated a value of 9,192,631,770 $\pm 20 \mathrm{c} / \mathrm{s}$ which is the best value available at present. The deceleration in the rotation of the earth about its axis as determined by the cesium standard is in the order of $1.16 \times 10^{-5}$ radians/year ${ }^{2}$, i.e., 0.16 seconds/year ${ }^{4}$, which is in good agreement with values obtained by the moon camera of 0.17 seconds/ year $^{2}$ for that period (June 1955 to June 1958). It is possible [Bullard, 1955] that Ephemeris Time and Atomic Time may not have the same rates; however, further careful experiments will be required to determine this. The stability of the best long time interval bases, which at present are quartz clocks steered by atomic standards is limited to values in the order of one part in $10^{10}$. Frequency comparisons quoted to higher precisions are relative, and may be made in two ways. One is to intercom. pare two oscillators directly for the short term, and the other is to compare one with the mean of a group of three or more of the same quality and assume that their variations are independent, so obtaining a statistical increase in precision for the group. The resonance of cesium is known in terms of the Ephemeris Second to an accuracy of only about 2 parts in $10^{9}$, the limitation being set by ${ }^{4}$ This means that if an atomic clock and "earth" clock were started together, at the end of a year the "earth" clock would be 0.08 sec behind the atomic clock. the uncertainty in the determination of the Ephemeris Second. Thus, high quality clocks closely steered by atomic standards will not realize the presently defined unit of time with any greater accuracy than this because of the nature of the definition itself. Presently, maximum available relative frequency stabilities for periods of several hours are in the order of: 1 part in $10^{11}$ for Crystal standards and 1 part in $10^{12}$ for the Ammonia Maser.

\section{Basic Limitations of Precision in the Fre- quency and Time Standards Via Radio Signals}

With the availability of very stable time references and frequency standards, it is obvious that considerable care must be exercised if these standards are to be distributed over large areas of the world without appreciable deterioration. If the terrestrial propagation of radio wave energy was at a constant velocity equal to the velocity of light in a vacuum and with a noise free background, it would be a relatively simple matter to distribute frequency and time signals with essentially no loss in stability and accuracy. Under these conditions, the received frequency would be unaffected and the time signals would have a given correctable constant delay of $T^{\prime}{ }_{d}=d / v_{0}$ where: $d$ is the distance and $v_{0}$ is the velocity of light $\simeq 3 \times 10^{5} \mathrm{~km} / \mathrm{s}$. In practice the radio energy is transmitted along a path with an effective velocity $(v)$ which varies with the characteristics of and conditions along the transmission path so that the actual delay, $T_{d}$, may vary also. It is obvious that the time indicated at a given receiving site is $T_{r}=T_{t}+T_{d}$, where the receiving clock can be made to indicate transmitted time $T_{t}$ by subtracting $T_{d}$ from the received time $T_{r}$ during clock setting. At the high frequencies, 2.5 to 30 $\mathrm{Mc} / \mathrm{s}$, presently employed for the distribution of frequency and time signals, multiple propagation paths frequently exist with appreciably different delays which can introduce large apparent errors in the received frequency and time. For any given path it may be possible by the proper choice of frequencies to have essentially one dominant mode of propagation; however, even a single mode may have appreciable variations in transmission time.

\subsection{Phase Distortion Limitations}

In figure 1 the straight dashed line represents the distortionless transmission in free space where the phase delay in radians could be given as

$$
\phi^{\prime}=\frac{2 \pi f d}{v_{0}}
$$

where: $f$ is the frequency in cycles per second, and $d$ is the path length expressed in kilometers.

The actual propagation medium, assuming one mode of multiple path to be dominant, may be considered as an electrical network with phase and amplitude versus frequency characteristics which vary as a function of time. The actual time 


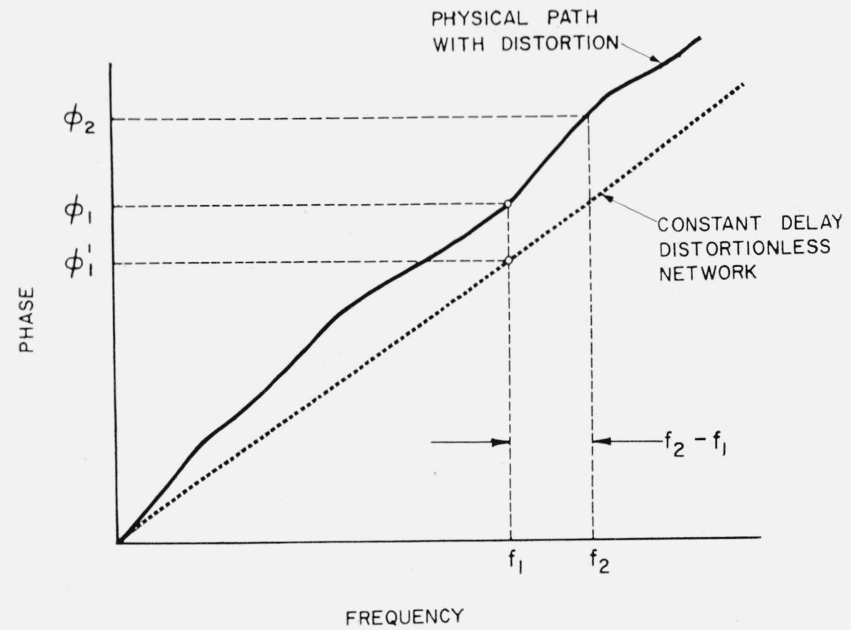

Figure 1. Transmission path phase characteristics.

delay is

$$
T_{d}=d / v_{p}
$$

where $v_{p}$ is the average path phase velocity in kilometers per second. It is well known in electrical circuit theory that various types of network delays exist, and if we are to consider the phase delay defined in eq (1), $v$ must be expressed in terms of path phase velocity rather than envelope velocity.

The actual path phase velocity relative to the velocity of light, $v_{p} / v_{0}$, is known to be a function of frequency and the path involved. Important factors may include ionospheric conditions, ground conductivity, and surface roughness. In the VLF region, Jean, Taylor, and Wait [1960] have shown good agreement between experimentally and theoretically determined values of $v_{p}$ over the 4 to 20 $\mathrm{kc} / \mathrm{s}$ frequency range. (See also Wait [1961a] and Wait and Spies [1961].) At night they have found values of $v_{p} / v_{0}$ to be $\simeq 1.03$ at $4 \mathrm{kc} / \mathrm{s}, 1.01$ at $10 \mathrm{kc} / \mathrm{s}$, and 1.003 at $20 \mathrm{kc} / \mathrm{s}$. Many references pertinent to the velocity of propagation are contained in the paper by Jean, Taylor, and Wait [1960]. In general, $v_{p}$ increases with surface conductivity, and decreases with increase in ionospheric height and earth's surface roughness.

An actual physical path phase characteristic may look somewhat like the solid wavy curve of figure 1 where a frequency $f_{1}$ would be delayed by the phase $\phi_{1}$. This phase delay in seconds can be written as

$$
T_{d}=\frac{\equiv \phi_{1}}{2 \pi f_{1}}
$$

where $T_{d}$ is the equivalent time delay in seconds. It should be pointed out that the particular phase versus frequency curve illustrated will only apply for a given distance and at a given time and that it may vary in its exact position as a function of time. The effect of these short term variations of phase as a function of time is to limit the precision with which a given standard frequency can be received.

To obtain the value of the received frequency

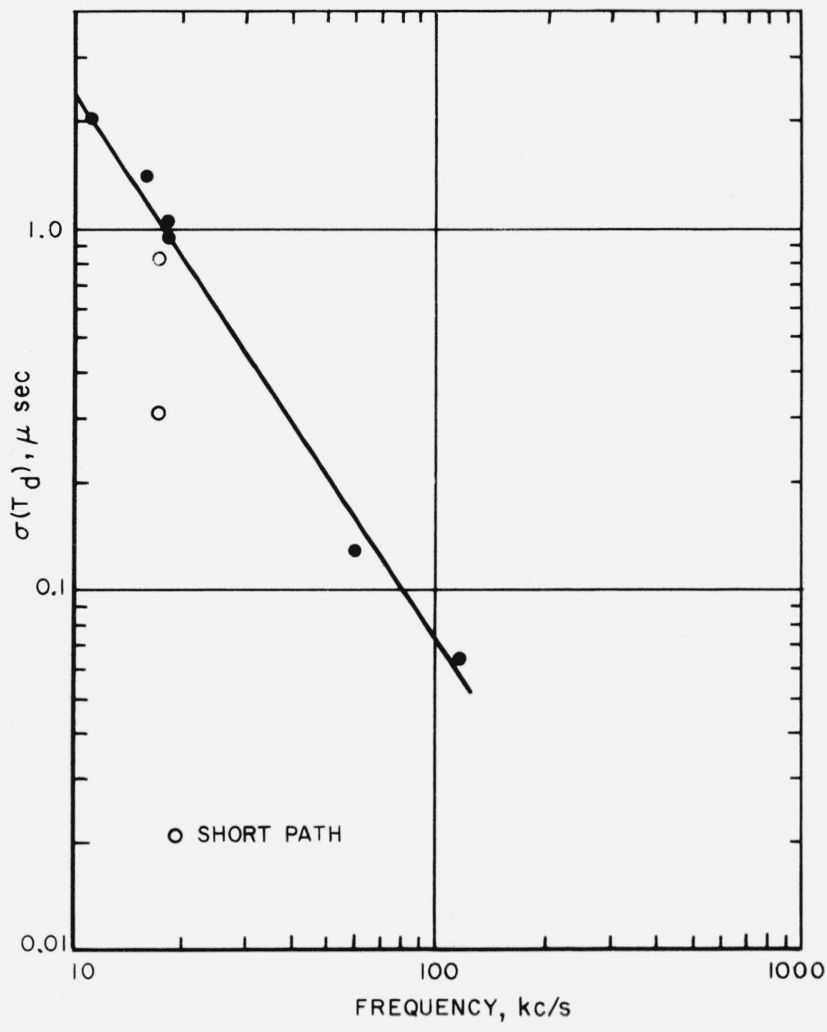

Figure 2. Short term propagation time (phase) stability daytime paths, normalized to single reflection sky wave.

from the phase measurements it is clear from the definition of frequency that the average departure from the transmitted frequency, $\overline{\Delta f}$, observed in terms of the final and initial phase delays is given by:

$$
\overline{\Delta f}=\frac{\phi(T)-\phi(0)}{2 \pi T}
$$

where $T$ is the time interval, in seconds, over which the phase comparison is made.

The relations governing the transmission path phase stability and signal-integration times required for specific frequency-comparison precisions have been described previously in considerable detail [Watt and Plush, 1959]. In general, where possible, observations should be made near noon at the center of the path. At such time, the phase variations, due to diurnal and random short term effects, are expected to be minimal. Pierce [1957] has experimental evidence over a $5200 \mathrm{~km}$ path that the total diurnal phase variation of the $16 \mathrm{kc} / \mathrm{s}$ Rugby signal is surprisingly constant throughout the year being in the order of $34 \pm 1 \mu$ sec. Jean ${ }^{5}$ over a $7500 \mathrm{~km}$ path has observed that the diurnal variation of the phase of the $16 \mathrm{kc} / \mathrm{s}$ Rugby signal has substantial variations in pattern. The observed average phase change of $\sim 42 \mu$ sec yields about the same effective change in ionospheric height; i.e., $\Delta \mathrm{h} \simeq 16 \mathrm{~km}$ using a method described by Wait [1959]. The diurnal patterns observed by Jean 
appear to change systematically with seasons and appreciably particularly during ionospherically disturbed conditions. The daytime transmission delay appears to be much less affected by these disturbances than the nighttime phase.

The short time variations in propagation time resulting from path phase instabilities can, in general, be assumed to be randomly distributed about an average time delay $\overline{T_{d}}$ with a rate of change similar to the fade rate. This means that a single measured value of the delay ranges roughly between $\bar{T}_{d} \pm \sigma\left(T_{d}\right)$, where $\sigma\left(T_{d}\right)$ is the standard deviation of the random time delay variable. In general, there will be different values for both $\bar{T}_{d}^{\prime}$ and $\sigma\left(T_{d}\right)$ for day or night paths. Expected standard deviations of short time propagation time delays are shown in figure 2 as a function of frequency for a single reflection daytime skywave in the VLF and $\mathrm{LF}$ regions. It should be emphasized that this trend does not continue up into the $\mathrm{HF}$ region. The data are obtained from table B-1 [Watt and Plush, 1959] with the additional point at $10.2 \mathrm{kc} / \mathrm{s}$ from Tibbals. ${ }^{6}$

\subsection{Time Reference and Clock Setting}

It is obvious that if a single precisely known uniform frequency were available at a receiving location, that it could be employed in the control of a time standard clock, provided: (a) that the clock could be properly set initially, and (b) that there were no interruptions in the frequency standard or clock. Since neither of these can be guaranteed, it becomes apparent that some method of establishing a time or phase reference at the receiving location is necessary, and that the problem of transmitting a precise time reference is much more

${ }^{6}$ M. L. Tibbals, private communication.

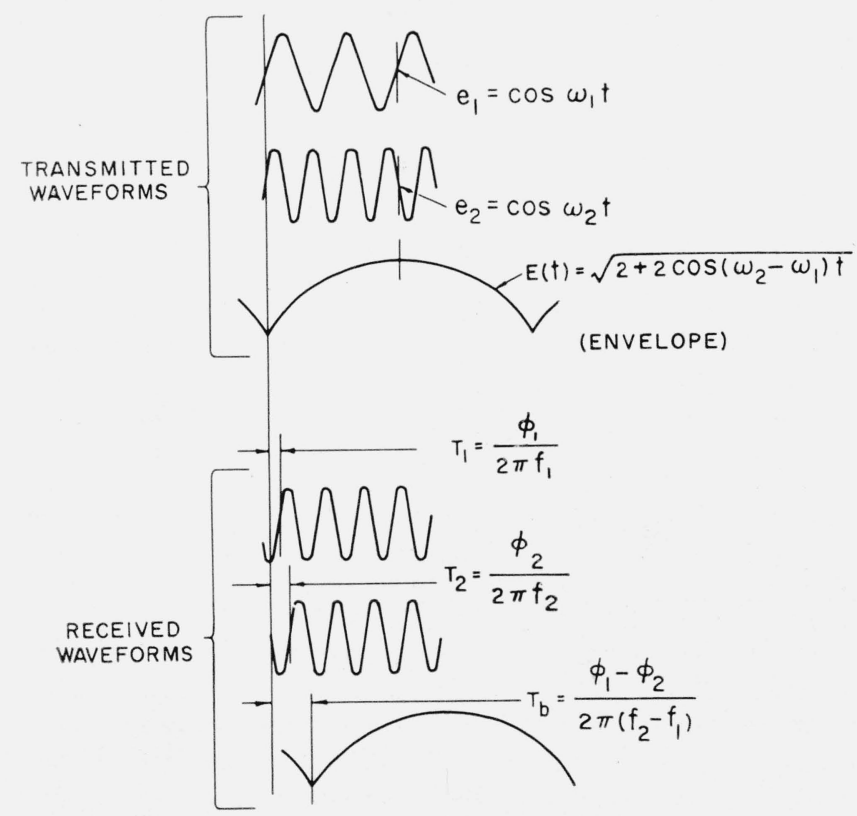

Figure 3. Standard frequency and time signal waveforms. difficult than a standard frequency transmission. At the higher frequencies where bandwidth limitations are not severe, this is accomplished by transmitting rather short pulses whose leading edges at the transmitter are quite steep. The delay of these time pulses is also variable but it is not the same as the phase delay described in eqs (2) and (3). Since the pulses are applied in the form of modulation on the rf carrier. the "envelope delay" as defined by Nyquist and Brand [1930] in terms of the slope of the phase characteristic, $d \phi / d f$, is the important factor. As we will see in the next section, the $\mathrm{HF}$ paths are very instable in both phase and envelope time delay and the timing accuracy obtainable with these pulses is considerably less than that available at the transmitter.

In view of the much more stable phase delay conditions in the VLF region as described by Pierce [1957] and Allan. Crombie, and Penton [1956] along with its excellent coverage characteristics, considerable interest has been exhibited in employing this medium for the distribution of standard frequencies and time reference. The channel bandwidths available in this frequency region, along with the very narrow bandwidths of the transmitting antenna, prevent the use of short-time pulses for the establishment of coarse time markers which could be used in identifying a particular rf cycle. An alternate method employing two closely spaced and alternately transmitted related frequencies is being considered. A description of this method is given by Morgan [1961]. The transmitted and received waveforms for such a narrow band time signal system are shown in figure 3 where it is apparent that the envelope of the voltage difference produces time reference markers with ambiguities spaced by the reciprocal of the frequency difference. Coarse time markers may, if desired, be provided by the times of frequency switching. On-off keying can also be considered for date time code. In a dual frequency system. transmitted waveforms are given as:

$$
\begin{aligned}
& e_{1}=\cos \omega_{1} t \\
& e_{2}=\cos \omega_{2} t
\end{aligned}
$$

while the envelope of the phasor sum is given as

$$
E(t)_{\text {envelope }}=\sqrt{2+2 \cos \left(\omega_{2}-\omega_{1}\right) t}
$$

It can be observed that at the transmitter, zero time is our phase reference. At the receiver, the phases of $e_{1}$ and $e_{2}$ are delayed and these two signals are

$$
\begin{aligned}
& e_{1 r}=\cos \left[\omega_{1} t+\phi_{1}\right] \\
& e_{2 r}=\cos \left[\omega_{2} t+\phi_{2}\right]
\end{aligned}
$$

Since these two signals can be employed to phase lock high quality local oscillators, it is possible to have two highly constant amplitude sinusoids as indicated. The envelope voltage waveform at the receiver is then

$E(t)_{\text {envelope received }}=\sqrt{2+2 \cos \left[\left(\omega_{2}-\omega_{1}\right) t+\left(\phi_{2}-\phi_{1}\right)\right]}$

where it is evident that the important phase is now 
$\phi_{2}-\phi_{1}$ and the resulting time delay is

$$
T_{b}=\frac{\phi_{2}-\phi_{1}}{2 \pi\left(f_{2}-f_{1}\right)}
$$

where $T_{b}$ is the envelope delay in seconds.

If the individual frequencies concerned are separated far enough, the path phase fluctuations become independent and the standard deviation of $T_{b}$ would be

$$
\sigma\left(T_{b}\right)=\frac{\sqrt{2} \sigma(\phi)}{2 \pi\left(f_{2}-f_{1}\right)}
$$

assuming that $\sigma\left(\phi_{1}\right)=\sigma\left(\phi_{2}\right)=\sigma(\phi)$.

It can be seen that the time variations will decrease with an increase in frequency separation. It should be emphasized that as the frequency separation is reduced the relative phases tend to become correlated and as a result the envelope reference time variation does not increase without limit since the phase difference tends to approach zero. Measured standard deviations of envelope delay variations for a $1 \mathrm{kc} / \mathrm{s}$ frequency spacing in the VLF band are shown by Casselman, Heritage, and Tibbals [1959] to be in the order of $\pm 20 \mu \mathrm{sec}$. (See also Stone, Markowitz, and Hall, 1960.) ${ }^{7}$

Employing the ionospheric roughness parameter described by Watt and Plush [1959] the standard deviation of short term phase variation; i.e., $\sigma(\phi)$, of a VLF carrier received over a $4200 \mathrm{~km}$ path is found to be in the order of 0.09 radians. When this value is used in (12) the expected $\sigma\left(T_{b}\right)$ expressed in microseconds is shown in figure 4. Experimental values for $\sigma\left(T_{b}\right)$ obtained from Heritage and Tibbals ${ }^{8}$ for spacing of $200,1.000$, and $3,000 \mathrm{c} / \mathrm{s}$ appear to substantiate the general level and trend of this curve.

In order to permit identification of a specific cycle of the carrier frequency $f_{1}$, it is obvious that $\sigma\left(T_{b}\right)$ must be less than $0.5 / f_{1}-\sigma\left(T_{d}\right)$ which at $20 \mathrm{kc} / \mathrm{s}$ will be in the order of $\pm 22 \mu$ sec. Until such time as the expected reduction in $\sigma\left(T_{b}\right)$ due to integration for time intervals long compared to the fade periods is obtained, it appears that frequency separations in the order of $1 \mathrm{kc} / \mathrm{s}$ will be required for carrier cycle identification in the $20 \mathrm{kc} / \mathrm{s}$ region. If the frequency separation chosen is rather large, the $\left(f_{2}-f_{1}\right)$ markers may be difficult to resolve from the on-off keying envelope. This is due in part to the normal build up time required by the narrow antenna bandwidths, see typical shapes in figure 5 , and the variation in position of this envelope as received due to path envelope delay. Should the on-off keying phase markers received with time delay variations $\sigma\left(T_{e}\right)$ not be stable enough to resolve $\left(f_{2}-f_{1}\right)$ markers, it may be necessary to employ an additional frequency providing an intermediate reference, viz $\left(f_{3}-f_{1}\right)<\left(f_{2}-f_{1}\right)$.

Since the precision of frequency comparison is defined as the phase jitter divided by the observing

7 Stone, Markowitz, and Hall in a recent paper [1960] have indicated VLF time comparisons of $500 \mu \mathrm{sec}$ at Washington, D.C., from NBA in Panama. Employing a $6 \mathrm{db}$ transmitting bandwidth of $44 \mathrm{c} / \mathrm{s}$ on fig. 4 and assuming that the envelope matching would approach that of two frequencies spaced by the $6 \mathrm{db}$ bandwidth we obtain $\sigma\left(T_{b}\right) \simeq 450 \mu$ sec for a path of comparable length. Actually the spacing chosen should likely be less which would yield better agreement. 8 Private communication. period $T$ (7.3 of Watt and Plush, 1959) we can write

$$
\epsilon=\sqrt{2} \sigma(\phi) / 2 \pi f T \text {. }
$$

The time delay for a given phase delay is $T_{d}=\phi / 2 \pi f$, and since $\sigma\left(T_{d}\right)=\sigma(\phi) / 2 \pi f$, we can write (13) as

$$
\sigma\left(T_{d}\right)=\epsilon T / \sqrt{2} .
$$

Figure 6 shows the manner in which this relationship can be employed to determine the variations expected in a given clock relative to a standard clock $T$ seconds after it has been set to coincidence with the standard clock. It is interesting to note that if an all daylight radio path such as the $16 \mathrm{kc} / \mathrm{s}$ transatlantic path observed by Pierce [1957] is employed, the maximum standard deviation of time difference is less than $2 \mu \mathrm{sec}$.

It should be pointed out that the problem of subtracting out the mean true transmission time, $\overline{T_{d}}$,

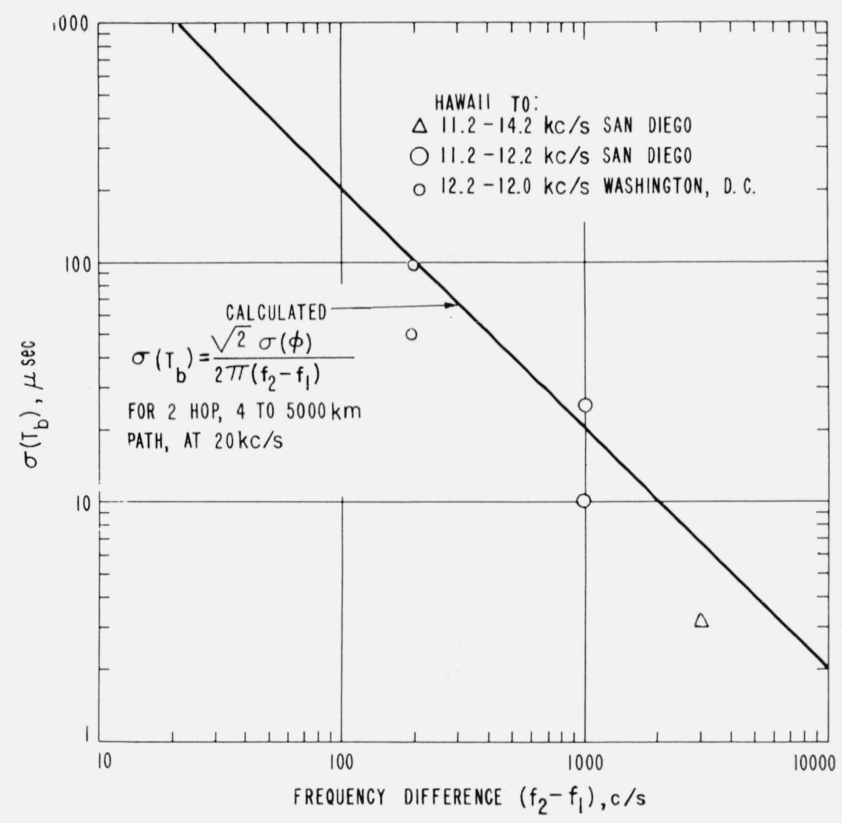

Figure 4. VLF transmission path envelope phase stability.

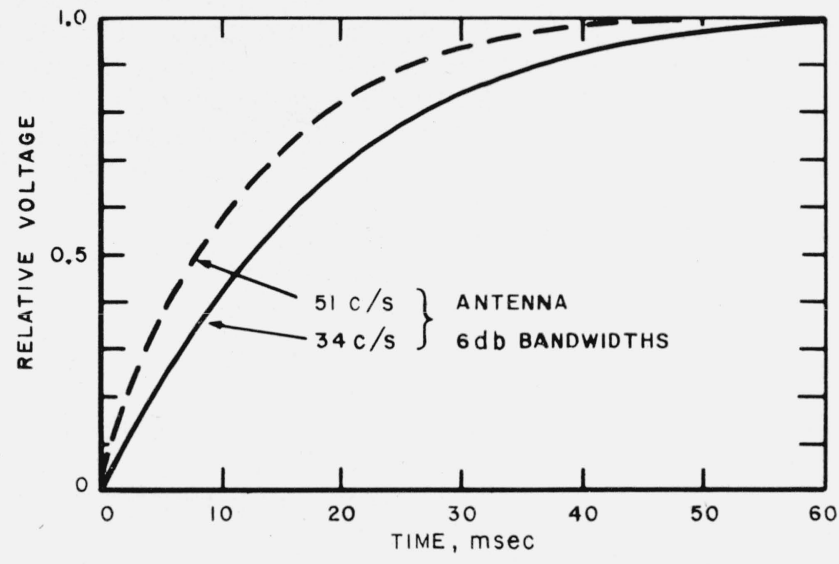

Figure 5. Keying response envelope of typical VLF transmitting antenna. 


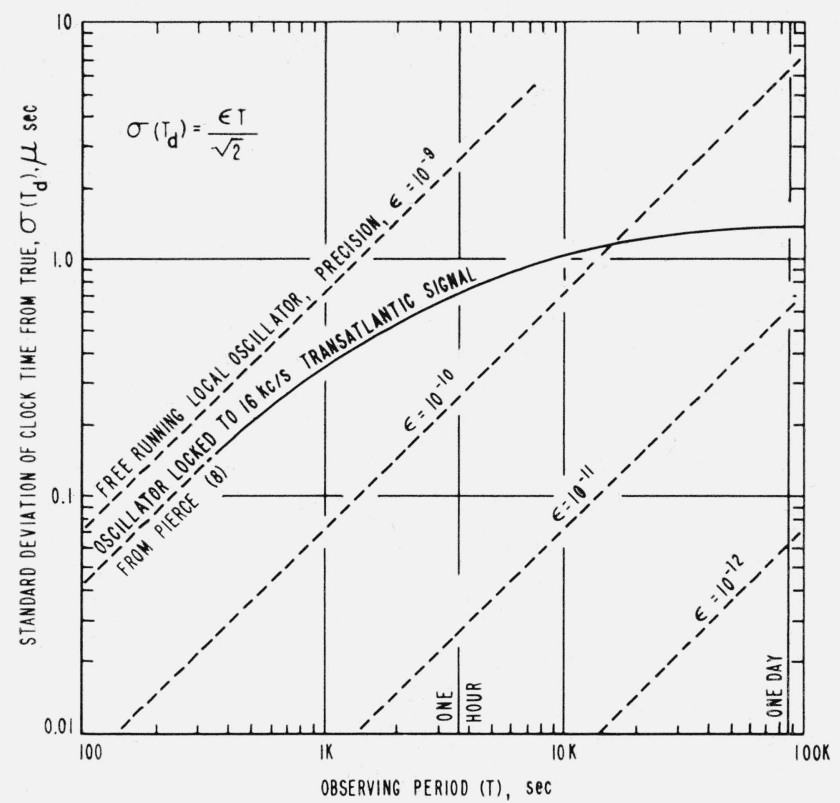

Figure 6. Clock-time deviations from original setting as a function of observing period. ( ${ }^{8}$ Pierce 1957).

whether it be a phase delay or envelope delay, still exists. Ionospheric disturbances caused by solar related proton storms or meteoric showers have the largest apparent effect on the nighttime phase delay [Chilton, 1961]. As a result, the midday values of $\overline{T_{d}}$ are expected to be relatively constant from day to day with the exception that solar flares effect the day values. Over some paths, it is possible that there may be appreciable seasonal variations in even the midday value of $\overline{T_{d}}$. Additional studies are required before the errors likely in predicting $\overline{T_{d}}$ can be specified as closely as desired. If two or possibly three VLF standard frequency and time signal stations are operating simultaneously at different $\mathrm{rf}$ frequencies but with a common frequency base as well as a common time reference, it is expected that the average delays due to transmission time can be obtained with greater accuracy. Once the average path delays have been subtracted out, a clock at a given receiving site can be set with a high degree of precision (probably limited mainly by the ability to determine absolute average path delay), to the time generated by the network of VLF standard frequency and time signal broadcasting stations.

\subsection{Carrier to Noise Limitations}

The limitations of noise to precision of frequency comparison have been previously described [Watt and Plush, 1959] and we shall only calculate the effects of rms carrier to rms thermal noise ratio on time signal accuracy.

From equation (C1) of Watt and Plush [1959] and (3)

$$
\underset{(\text { noise })}{\sigma\left(T_{d}\right)}=\frac{N / C}{2 \sqrt{2} \pi f_{1}}
$$

where $\sigma\left(T_{d}\right)$ (noise) is now the standard deviation of the time reference in seconds derived from the carrier frequency $\left(f_{1}\right)$ phase, and $N / C$ is the rms noise to carrier ratio in the receiver. With post detection filtering or integration following a linear detector, $N$ should be calculated in the effective bandwidth of the integrator. If we consider noise density or in particular, the noise voltage per $1 \mathrm{kc} / \mathrm{s}$ bandwidth concept where $\mathrm{N}_{1 \mathrm{kc}}$ has the dimensions volts $/ \sqrt{\mathrm{kc} / \mathrm{s}}$, we obtain

$$
\underset{(\text { noise })}{\sigma\left(T_{d}\right)}=\frac{\sqrt{B_{r} / 1,000}}{2 \sqrt{2} \pi f_{1} C / N_{1 \mathrm{kc}}}
$$

where $B_{r}$ is the effective receiver bandwidth in cycles per second.

When timing is derived by an envelope beat pattern between two carriers, the effects of noise are given by

$$
\underset{(\text { noise })}{\sigma\left(T_{b}\right)}=\frac{N / C}{2 \pi\left(f_{2}-f_{1}\right)}
$$

or

$$
\underset{(\text { noise })}{\sigma\left(T_{b}\right)}=\frac{\sqrt{B_{r} / 1,000}}{2 \pi\left(f_{2}-f_{1}\right) C / N_{1 k c}}
$$

where it is obvious that the time stability for these conditions is poorer for a given $C / N_{1 \mathrm{kc} / \mathrm{s}}$ ratio than obtains for $\sigma\left(T_{d}\right)$ (noise).

We can also determine the stability of a time reference obtained from a keyed envelope obtained at the one half amplitude point of the leading edge with coherent detection. For a single sample this is shown in the appendix to be

$$
\sigma\left(T_{e}\right)=\frac{1}{(C / N) 2 B_{t}}
$$

where: $\sigma\left(T_{e}\right)$ is in seconds and $B_{t}$ is the transmitter $6 \mathrm{db}$ bandwidth in $\mathrm{c} / \mathrm{s}$. If the carrier is keyed on periodically (for example, once each second) the resulting envelope reference can be integrated to reduce the effects of noise. The improvement is equal to the square root of the number of individual pulses, and if the repetition rate is $F$

$$
\sigma\left(T_{e}\right)=\frac{1}{(C / N) B_{t} \sqrt{T F}}
$$

or

$$
\sigma\left(T_{e}\right)=\frac{\sqrt{B_{r} / 1,000 T F}}{\left(C / N_{1 k c}\right) B_{t}}
$$

where: $T$ is the integration period, $T F$ is the number of samples averaged, $B_{r}$ is the effective receiver bandwidth in c/s. It is apparent from (21) that the time reference derived from the envelope leading edge improves directly as the carrier to noise ratio and as the first power of the transmitting antenna bandwidth and as the square root of the 
integration time. In all the derivations involving the rms carrier to noise ratios, the significant noise background considered is of thermal type. The actual background will normally be atmospheric noise which in a wide bandwidth has a very different statistical nature [Watt and Maxwell, 1957]. The effects of atmospheric noise upon radio systems are in general quite different from those of thermal noise [Watt, Coon, Maxwell, and Plush, 1958]. For a pulse sampling system with integration, the required rms $C / N$ may be appreciably less than indicated in eqs. (20) and (21). Hefley ${ }^{9}$ [1960] for example has found with Loran-C systems that for equivalent performance the carrier to atmospheric noise ratio can be much less than the carrier to thermal noise ratio; however, it must be remembered that this system employs a very wide RF bandwidth.

When carrier phase or frequency stability is considered, the narrow bandwidths $(0.1 \mathrm{c} / \mathrm{s}$ or less $)$ employed will make the atmospheric noise statistics the same as thermal noise [Watt and Maxwell, 1957] and in these cases the formulas given (15) through (18) should apply if linear receivers are employed.

\section{Present Services}

There are at present quite a large number of standard-frequency and time-signal stations in use throughout the world. VLF, LF, and HF are employed for large area coverage with some higher frequencies being used for local distribution. A description of the stations operating along with their location and characteristics is given in Annex I of CCIR Report 66 [1956].

Annex II of the same document describes the characteristics of projected standard frequency and time signal stations at four additional locations. In general, the high frequency stations all employ pulse modulation of the carrier frequency for time signal transmissions and many of them in addition have standard audiofrequencies employed as modulation at various times. The stability as broadcast is usually very good, for example with the National Bureau of Standards station WWV [1960a] the carrier and audiotones have stability of one part in $10^{9}$ at all times with normal daily deviations of less than two parts in $10^{10}$. The time signals have essentially the same stability and the maximum deviation from U'T-2 is about \pm 50 msec. Final corrections to the frequency as broadcast versus the U.S. Frequency Standard are published monthly by the National Bureau of Standards [1960b] in the Proceedings of the IRE while final corrections to the time signals versus U'T-2 time are published by the U.S. Naval Observatory.

\subsection{HF Standard Frequency and Time Services}

Because of the variation in the propagation medium at $\mathrm{HF}$, the frequency as received is generally much less stable than that transmitted. Actually, for high precision measurements it is frequently necessary to use long averaging periods

${ }^{9}$ Private communication. of 10 to 30 days. Ideally, the following conditions should prevail at the time measurements are being made; (a) all daylight (or darkness) over radio path, (b) no ionospheric disturbance in progress, (c) no part of the radio path should pass near either auroral zone, (d) single mode propagation should be existing. In general, frequency determinations can be made either by a direct comparison or by means of the time pulses. Some idea of the precision of frequency comparison obtainable can be seen from figure 7 which shows the precisions available from WWV and WWVH over paths ranging from approximately 2,000 to $8,000 \mathrm{~km}$. Line A shows that for short period samples, such as $100 \mathrm{sec}$, the precision available is in the order of 2 parts in $10^{7}$. If similar 100 -sec samples are made each day, after 30 days the precision has improved to one part in $10^{8}$. It should be emphasized that this type of frequency comparison does not make full use of the transmissions and that the improvement with observing period is not as great as would be predicted if all the actual information received was fully utilized for the total periods shown. Line B shows the results obtained at Boulder employing time pulses compared for approximately 10 sec near noon each day and averaged by means of a clock. Here the daily averaging time is $24 \mathrm{hr}$. The point shown for 30 days is arrived at as follows. Daily comparisons of the local clock versus the received time of $\mathrm{WWV}$ are used to obtain running 10-day averages of time difference. Each day one new daily value is added and the 11-days old value is dropped and the re-

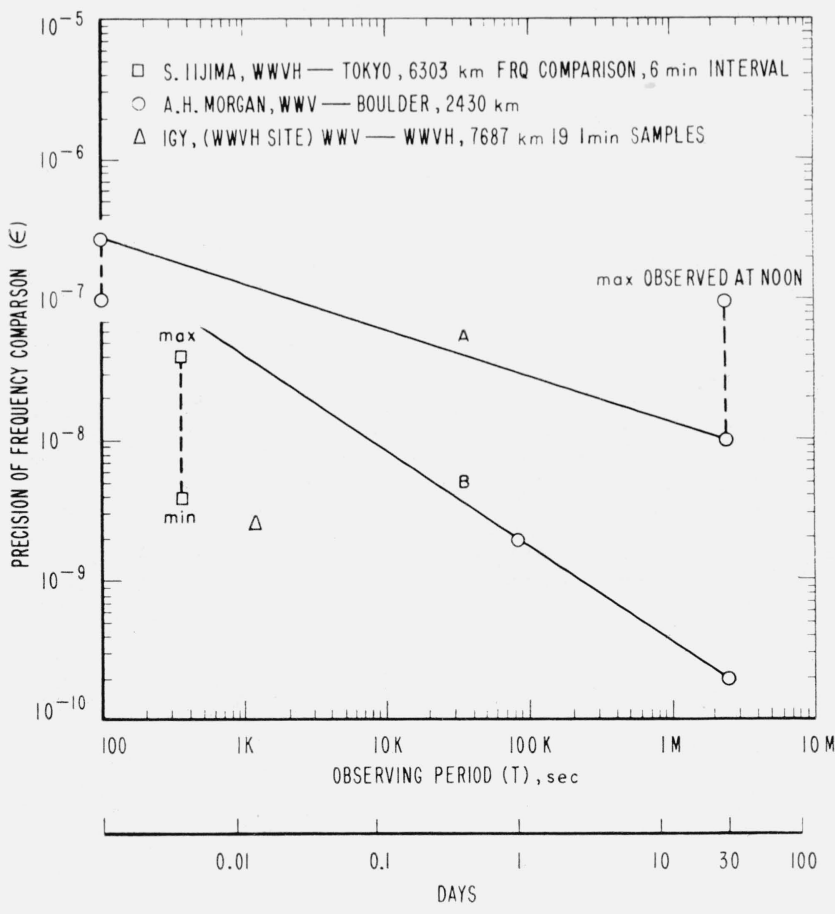

Figure 7. Frequency comparison precision at $10 \mathrm{Mc} / \mathrm{s}$. Line $A=100$ sec samples, frequency comparisons 1 sample/day at noon. Line $B=24$-hr average each day, time pulses, compared at 10 a.m. local time. All values are standard deviation and at best time of day (disturbed day data not included). 
sultant 10 time differences averaged. The 30-day running averages of the above 10-day averaged values are obtained in a similar manner. The precisions obtained are appreciably better, being in the order of \pm 2 parts in $10^{9}$ for 1 day and approximately \pm 2 parts in $10^{10}$ for 30 days.

\subsection{LF Standard Frequency Transmissions}

At present there are at least six low frequency standard transmissions being operated including MSF at $60 \mathrm{kc} / \mathrm{s}$ in Rugby, England; WWVB at $60 \mathrm{kc} / \mathrm{s}$ in Boulder, Colo.; DCF77 at $77.5 \mathrm{kc} / \mathrm{s}$ in Mainflingen, Germany; OLP at $50 \mathrm{kc} / \mathrm{s}$ in Czechoslovakia; HBJ at $96.04 \mathrm{kc} / \mathrm{s}$ in Switzerland; and A5XA at $1331 \frac{1}{3} \mathrm{kc} / \mathrm{s}$ at Fort Monmouth, N.J., U.S.A. Other characteristics of these transmissions are described in Annex III of CCIR Report 66 [1956]. Pierce [1957] has shown the standard deviation of frequency comparison for MSF transmissions on a transatlantic path to be in the order of 1 times $10^{-10}$ for a 30 -minute observing period. Further calculations for LF transmissions are compared with experimental results by Watt and Plush [1959] and shown here in figure 8 . Pierce [1958] has shown. average frequency comparison precisions from WWVB $(60 \mathrm{kc} / \mathrm{s})$ as received at Cruft Laboratory, of 1.4 parts in $10^{11}$ in an observing period of about one hr. From equation (14) this would correspond to a short term time variation $\sigma\left(T_{d}\right) \simeq 0.36 \mu$ sec.

Recent plans to distribute time signals via Loran-C navigation system, pulse transmissions at $100 \mathrm{kc} / \mathrm{s}$, have been described by Doherty [1961]. Very precise time signal distribution in the order of $0.2 \mu$ sec is possible within the ground wave coverage range of about $1,500 \mathrm{~km}$. By employing sky wave modes,

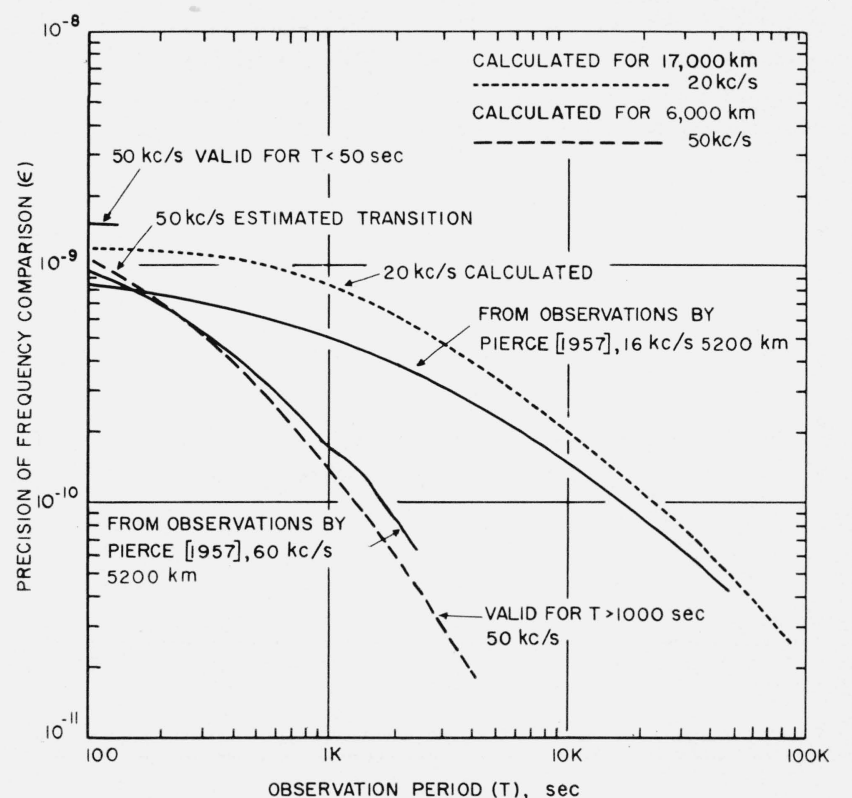

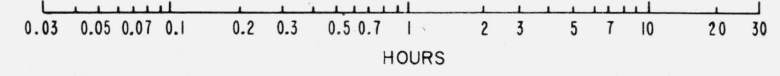

FiguRE 8. Experimental and theoretical precisions. Note: Due to diurnal phase change observing periods of longer than $10 \mathrm{~K}$ nclude several days. timing precision in the order of $1 \mu$ sec appears likely out to about $3,500 \mathrm{~km}$.

It is obvious from this and the preceding sections that in a limited area the LF systems (50 to 100 $\mathrm{kc} / \mathrm{s}$ ) will provide for more precise comparison of frequency and time than the VLF systems (15 to $20 \mathrm{kc} / \mathrm{s}$ ). The propagation loss, however, is much higher in the 50 to $100 \mathrm{kc} / \mathrm{s}$ band which restricts the useful range to distances of about 3,000 to 4,000 $\mathrm{km}$. LF transmissions at $60 \mathrm{kc} / \mathrm{s}$ in England [Steele, 1955] have shown that timing can be obtained to $\pm 15 \mu \mathrm{sec}$ by pulse envelope methods.

\subsection{VLF Standard Frequency Transmissions}

Annex III of CCIR Report 66 [1956] describes the transmissions from GBR at $16 \mathrm{kc} / \mathrm{s}$ which has been used as a basis for much of the pioneer work on standard frequency broadcasting in the very low frequency region. The precisions available from this transmission, and GBZ on $19.6 \mathrm{kc} / \mathrm{s}$, based primarily on observations by Pierce $[1957,1958]$ and Allan, Crombie and Penton [1956], are included in sections 2.2 and 4 . Stabilized transmissions with phase locked master-slave relationship from Hawaii and San Diego have also served as a valuable source of information for VLF path phase stability as described by Casselman, Heritage, and Tibbals [1959]. Recently interim station broadcasts of the U.S. frequency standard have been initiated from WWVL on $20 \mathrm{kc} / \mathrm{s}$ from near Boulder, Colo. Time and stabilized frequency broadcasts from NBA at $18 \mathrm{kc} / \mathrm{s}$ also recently have been initiated. ${ }^{10}$ Additional VLF transmissions are in the process of being stabilized in frequency which should make them available for path phase stability measurements. In the VLF region both theory and experiment show that greater observing times are required for a given degree of frequency comparison precision than is true in the LF region; however, when long range paths are considered the low attenuation rates obtaining in the VLF band make emissions in this frequency region useful for worldwide coverage.

\section{Characteristics and Expected Coverage for a VLF Standard Frequency and Time Signal Broadcasting Station}

Based on the previous analysis [Watt and Plush, 1959] the precision of frequency comparison expected as a function of observing period $T$ was prepared and is shown as figure 8 [Pierce, Winkler, and Corke, 1960]. ${ }^{11}$ It is obvious when comparing figures 7 and 8 that a VLF broadcast will provide one or two orders of magnitude improvement over present $\mathrm{HF}$ broadcasts in precision of frequency comparison for a given observing period. This great increase in performance has clearly indicated the desirability of designing and constructing a high quality VLF standard frequency broadcasting station or network of 2 or 3 stations whose primary

10 Precise time and stabilized frequency broadcasts at $18 \mathrm{kc} / \mathrm{s}$ over NBA, Summit, Canal Zone, were announced in U.S. Naval Observatory Time Service, Notice No. 8, 18 November 1959. See also a recent paper by Stone, Markowitz, and Hall [1960].

A recent paper by Pierce, Winkler, and Corke [1960] describes results at 16 $\mathrm{kc} / \mathrm{s}$ over a $5,200 \mathrm{~km}$ path that indicate possible precisions of $2 \times 10^{-11}$. 
function is to provide extremely precise standard frequency and time signals.

Because of the desirability of having such a station near the U.S. standard of frequency located at Boulder, Colo., a study has been made to determine design parameters and service obtainable at various locations. Seventeen different receiving sites were chosen and the total number of measurements obtainable each day for 99 percent of all hours were calculated based on a radiated power of $100 \mathrm{kw}$. Calculations were made for a range of radiated powers from 25 to $100 \mathrm{kw}$ and the resulting number of measurements obtainable as a function of the cost per measurement was determined. The results of this study [Watt and Plush, 1959] indicated a preference for a radiated power in the order of $100 \mathrm{kw}$.

The results of this study are summarized in table 1 , where the various assumptions made are indicated along with a tabulation of the total number of measurements obtainable for 99 percent of all hours with $100 \mathrm{kw}$ radiated at $20 \mathrm{kc} / \mathrm{s}^{12}$ for the months of June and December assuming a precision of frequency comparison of 1 part in $10^{9}$. The method of analysis and some of the assumptions made in this analysis are contained in the following sections.

\subsection{Propagation Path Attenuation Rates}

It is relatively well known that the attenuation rate $(a)$ to be employed in field strength equations such as (2) in the paper by Watt and Plush [1959], expressed in decibels per $1,000 \mathrm{~km}(\mathrm{db} / \mathrm{K})$, is dependent upon a number of factors including: frequency, ionospheric conditions, earth's surface conditions, and the earth's magnetic field.

Experimental and theoretical studies indicate that there is a broad minimum in the attenuation-versusfrequency curve which is centered somewhere in the vicinity of 16 to $18 \mathrm{kc} / \mathrm{s}$. The shape of this attenuation curve is likely to vary between day and night conditions, and is also likely to be different for east-to-west and west-to-east propagation. It would appear from an analysis of results obtained from many different sources that the east-to-west (magnetic) attenuation rate in some cases may be as much as 1 or $2 \mathrm{db} / \mathrm{K}$ greater than the west-to-east attenuation rates. This effect is expected to be more pronounced at night; in addition this difference in attenuation rate with direction is expected to be greater at the lower frequencies; viz, 10 to $14 \mathrm{kc} / \mathrm{s}$, and relatively small in the vicinity of $20 \mathrm{kc} / \mathrm{s}$. These effects have been discussed by various authors [H. J. Round, T. E. Eckersley, K. Tremellen, and F. C. Lunnon, 1925; K. G. Budden, 1951 and $1952 ;$ J. R. Wait, 1958 and $1961 \mathrm{~b}$; and D. D. Crombie, 1958].

Approximate values for the average daytime attenuation rate $(a)$ in the vicinity of $20 \mathrm{kc} / \mathrm{s}$ are: sea $\simeq 2$, average land $\simeq 4$, estimated values for permafrost and icecap are 7 and 18 . When mixed surface conditions are encountered along the path, as is

$12 \mathrm{It}$ should be noted that $20 \mathrm{kc} / \mathrm{s}$ has been employed in these calculations because: (1) $20 \mathrm{kc} / \mathrm{s}$ is an internationally assigned standard frequency, (2) the power requirements for world wide coverage are expected to be near a minimum at this frequency, (3) considerable propagation data is available in this region. Further research on attenuation and phase stability should be conducted over the whole VLF spectrum to determine the suitability of other frequencies for standard frequency and time distribution. usually the case in practice, the total average path attenuation will not always be exactly equal to the sum of the attenuations expected from each individual portion of the path. This may be due in some cases to a transfer of energy from the dominant to higher order modes caused by the discontinuities in conductivity at the surface. It is also likely that low conductivity at the earth's surface will produce appreciably greater attenuation during the day when the ionosphere is low than at night when it is higher.

After determining the various amounts of surface types along the individual paths, expected average attenuation rates have been assigned for each of the receiving locations in table 1 . In some cases reciprocal path values have been employed and, in general, values quoted are as high as anticipated from a consideration of all possible attenuation mechanisms.

Field strengths anticipated for these particular paths have been calculated employing the relationships given by Watt and Plush [1959]; the results are shown in column 5 of table 1 for an assumed radiated power of $100 \mathrm{kw}$ at a frequency of $20 \mathrm{kc} / \mathrm{s}$.

\subsection{Expected Availability and Precision of Fre- quency Comparison}

The carrier to noise available at the receiving location may in some cases be the limiting factor as regards the time required to obtain a frequency comparison of, say, 1 part in $10^{9}$. Median atmospheric noise fields expected for the various receiving locations have been obtained from Crichlow, Smith, Morton, and Corliss [1955] and the results presented in columns 6 and 7 of table 1 . Combining the pre. vious information regarding carrier field intensities with the noise field intensity data, we have obtained the minimum expected observation times required to compare frequency to 1 part in $10^{9}$ for 100 kilowatts radiated as shown in columns 14 and 15 .

Pierce [1957] has shown that the transmission path phase is very stable and that the diurnal variations caused by sunrise or sunset along the Rugby to Cambridge path are surprisingly constant throughout the year. During the all sunlight period the path phase appears more stable than at night for frequencies of 16 to $60 \mathrm{kc} / \mathrm{s}$. We have determined, on the basis described by Watt and Plush [1959], the minimum observing times required to obtain a precision of 1 part in $10^{9}$ based on path phase variations expected for both day and night conditions. It should be noted that $\sigma(\phi)$ has been assumed to increase as the square root of distance which is what one would expect of sections of variable delay wave guides connected in series. These results are presented in columns 12 and 13.

Although it is obvious that in a case where minimum observing times based on path phase variability and carrier-to-noise ratio are nearly the same, the combined effect will require a longer observation time than indicated for either limitation. We have neglected this effect and have considered only the one condition which requires the longer period.

In order to get some idea of the relative service obtainable from the proposed standard frequency and time signal broadcast, the total number of 


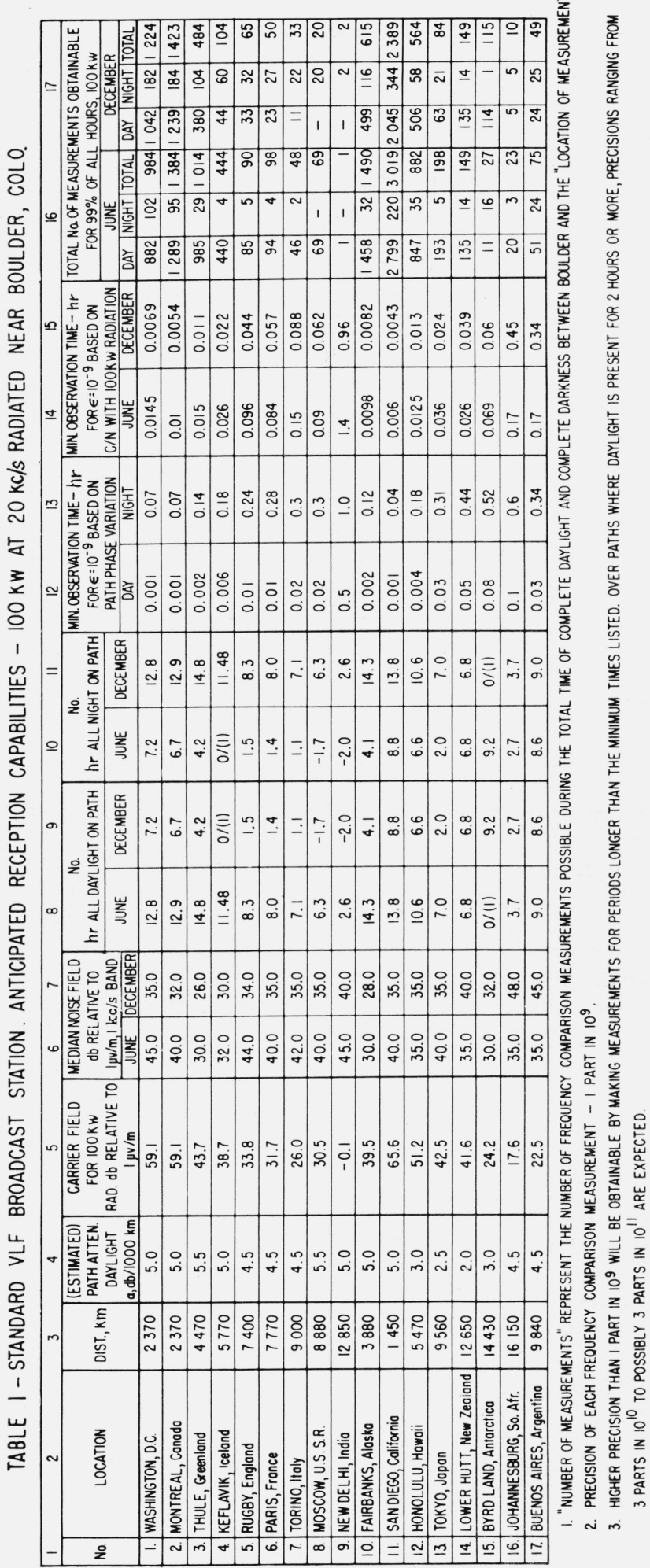

measurements with a precision of 1 part in $10^{9}$ obtainable per $24 \mathrm{hr}$ for 99 percent of all hours have been obtained by dividing the number of hours of all-day or all-night over the particular path by the appropriate minimum observing times and adding, as shown in columns 16 and 17 for the months of June and December. It can be seen from this tabula-tion that the service provided at various locations is appreciably different; however, ic is interesting to observe that in all but one case an appreciable number of measurements can be made throughout each day, and that in the worst case, for transmissions to New Delhi, at least one comparison with a precision of 1 part in $10^{9}$ is expected in each day. In many of the closer more favorable locations, it would appear that precision of 3 parts in $10^{10}$ or better can be expected where observing times of at least $2 \mathrm{hr}$ or more over daylight paths are possible. The maximum precision obtainable that can be expected and any locations can readily be calculated using the procedures outlined earlier [Watt and Plush, 1959].

\section{Appendix}

\subsection{Timing Âccuracy Obtainable With an On-Off Keyed Carrier in the Presence of Thermal Noise}

It is well known that the slope of the envelope at the one-half amplitude point of a keyed carrier passed through a filter with a $6 \mathrm{db}$ bandwidth $B_{t}$ is

$$
\frac{d v}{d t} \simeq E B_{t}
$$

where $E$ is the maximum or locked keyed carrier voltage, and $d v / d t$ is the slope observed at the onehalf carrier envelope amplitude point.

When thermal type noise is present, the envelope of the keyed carrier can be expected to have a standard deviation in amplitude of $\sigma(a)$ for $C / N \gg 1$ with an envelope detector or for all $C / N$ ratios if a synchronous detector is employed. If the point at which the noise free keyed carrier crosses the $E / 2$ point is chosen as a reference $T_{0}$, it can be seen that the actual crossing point in the presence of noise will vary about this point. The standard deviation of this crossing time is defined as $\sigma\left(T_{e}\right)$, and it is easily seen that

$$
\begin{gathered}
\sigma(a) / \sigma\left(T_{e}\right)=d v / d t=E B_{t} \\
\sigma\left(T_{e}\right)=\sigma(a) / E B_{t}
\end{gathered}
$$

Since $E=\sqrt{2} C$ where $C$ is the rms carrier and $\sigma(a)=\sqrt{2} N$ where $N$ is the rms noise in the receiver IF band pass filter, we can write

$$
\sigma\left(T_{e}\right)=\frac{1}{(C / N) B_{t}}
$$

When the receiver effective bandwidth is $B_{r}(\mathrm{c} / \mathrm{s})$ and we use the convention of $C / N_{1 k c}$, i.e., the rms carrier to rms noise in a one $\mathrm{kc} / \mathrm{s}$ effective band, we 
obtain for a single sample

$$
\sigma\left(T_{e}\right)=\frac{\sqrt{B_{r} / 1,000}}{\left(C / N_{1 k c}\right) B_{t}}
$$

The authors have benefited from many helpful discussions with numerous individuals. In particular, we would like to acknowledge information and suggestions obtained from J. R. Wait, J. A. Pierce, A. G. Jean, R. C. Kirby, W. Markowitz, K. A. Norton, J. M. Richardson, W. D. George, D. H. Andrews, and D. D. Crombie. The assistance of Mrs. Winifred Mau in the preparation of this manuscript is also gratefully acknowledged.

\section{References}

Allan, A. H., D. D. Crombie, and W. A. Penton, Frequency variations in New Zealand of $16 \mathrm{kc} / \mathrm{s}$ transmissions from GBR Rugby, Nature 17\%, 178 (1956).

Budden, K. G., The reflection of very low frequency radio waves at the surface of a sharply bounded ionosphere with superimposed magnetic field, Phil. Mag. 42, 833-851 (1951).

Budden, K. G., The propagation of a radio atmospheric-II, Phil. Mag. 43, 1179-1201 (1952).

Bullard, E. C., Definition of the second of time, Nature 176, 282 (Aug. 1955)

Casselman, C. J., D.P. Heritage, and M. L. Tibbals, VLF propagation measurements for the Radux-Omega navigation system, Proc. IRE 47, No. 5, 829-839 (May 1959).

CCIR VIII Plenary Assembly, Vol. I, Report 66, p. 362 (Warsaw, 1956)

Chilton, Charles J., VLF phase perturbation associated with meteor shower ionization, J. Geophys. Research 66, No. 2, 379-383 (Feb. 1961).

Crichlow, W. Q., D. F. Smith, R. N. Morton, and W. R. Corliss, World-wide radio noise levels expected in the frequency band from $10 \mathrm{kc}$ to $100 \mathrm{Mc}$, U.S. Dept. of Commerce, NBS Circ. 557 (Aug. 1955). (U.S. Govt. Printing Office, Washington 25, D.C.) See ITU, Geneva (1957).

Crombie, D. D., Differences between east-west and west-east propagation of VLF signals over long distances, J. Atmospheric and Terrest. Phys. 12, 110-117 (1958).

Doherty, R. H., G. Hefley, R. F. Linfield, Timing potentials of Loran-C, to be published in Proc. IRE (1961).

Jean, A. G., W. L. Taylor, and J. R. Wait, VLF phase characteristics deduced from atmospheric waveforms, J. Geophys. Research 65, 907-912 (March 1960).

Lyons, H., Spectral lines as frequency standards, Annals, N.Y. Academy of Sciences, p. 831 (May 1952).

Mach, Ernst, Science of mechanics, 5th English ed., p. 272 (Open Court Publishing Co., LaSalle, Ill., 1942).

Markowitz, William, Astronomical and atomic times, U.S. Naval Observatory, Washington 25, D.C. (9 March 1959). See also: Time measurement, Encyclopedia Britannica 22, 224-228 (1959).
Markowitz, W., R. G. Hall , L. Essen, and J. V. L. Parry, Frequency of cesium in terms of ephemeris time, Phys. Rev. Letters 1, No. 3, 105-107 (Aug. 1, 1958).

Morgan, A. H., Proposal for a new method of time signal modulation of VLF carriers (to be published as an NBS Tech. Note, 1961).

National Bureau of Standards, Boulder Laboratories, Standard frequency and time signals, WWV and WWVH, NBS Misc. Publ. 236 (1960).

National Bureau of Standards, Boulder Laboratories, National standards of time and frequency in the United States, Proc. IRE 48, No. 1, p. 105 (Jan. 1960).

Nyquist, H., and S. Brand, Measurement of phase distortion, Bell System Tech. J. IX, 522-543 (July 1930).

Pierce, J. A., Intercontinental frequency comparison by VLF radio transmission, Proc. IRE 45, 794 (1957).

Pierce, J. A., Recent long distance frequency comparisons, IRE Trans. on Instrumentation I- $\boldsymbol{\gamma}$, pp. 207-210 (Dec. 1958)

Pierce, J. A., G. M. R. Winkler, and R. L. Corke, The 'GBR experiment': A trans-Atlantic frequency comparison between caesium-controlled oscillators, Nature 187, 914-916 (July-September 1960).

Round, H. J., T. E. Eckersley, K. Tremellen, and F. C. Lunnon, Report of measurements made on signal strength at great distances during 1922 and 1923 by an expedition sent to Australia, J. Inst. Elec. Engrs. (London) 63, No. 346, 933-1011 (Oct. 1925).

Steele, J. McA. The standard frequency monitor at the $\mathrm{Na}$ tional Physical Laboratory, Proc. Inst. Elec. Engrs. (London), Part B, 102, 155-165 (1955).

Stone, R. R., Jr., W. Markowitz, and R. G. Hall, Time and frequency synchronization of Navy VLF transmissions, IRE, Trans. on Instrumentation I-9, No. 2, 155-161 (Sept. $1960)$

Wait, J. R., A study of VLF field strength data, both old and new, Geofisica pura e applicata 41, 73-85 (1958/III).

Wait, J. R., Diurnal change of ionospheric heights deduced from phase velocity measurements at VLF, Proc. IRE 47, 998 (1959).

Wait, J. R., A comparison between theoretical and experimental data on phase velocity of VLF radio waves, Proc. IRE 49, 1089-1090 (June 1961).

Wait, J. R., A new approach to the mode theory of VLF propagation, J. Research NBS (Radio Prop. 65D, No. 1, 37-46 (Jan.-Feb. 1961)

Wait, J. R., and K. P. Spies, A note on phase velocity of VLF radio waves, J. Geophys. Research 66, 992-993 (March 1961).

Watt, A. D., and E. L. Maxwell, Measured statistical characteristics of VLF atmospheric radio noise, Proc. IRE 45, 55-62 (Jan. 1957).

Watt, A. D., R. M. Coon, E. L. Maxwell, and R. W. Plush, Performance of some radio systems in the presence of thermal and atmospheric noise, Proc. IRE 46, No. 12, 1914-1923 (Dec. 1958)

Watt, A. D., and R. W. Plush, Power requirements and choice of an optimum frequency for a world-wide standard-frequency broadcasting station, J. Research NBS (Radio Prop.) 63D, No. 1, 35-44 (July-Aug. 1959).

(Paper 65D6-164) 